\title{
One-Pot Synthesis and Characterization of Size-Controlled Bimagnetic FePt-Iron Oxide Heterodimer Nanocrystals
}

\author{
Albert Figuerola, ${ }^{*, t, \ddagger}$ Angela Fiore, ${ }^{\dagger, \ddagger}$ Riccardo Di Corato, ${ }^{\ddagger}$ Andrea Falqui,, \\ Cinzia Giannini," Edoardo Micotti, ${ }^{\perp}$ Alessandro Lascialfari, ${ }^{\perp, \#}$ Maurizio Corti, ${ }^{\perp}$ \\ Roberto Cingolani, ${ }^{\dagger, \ddagger}$ Teresa Pellegrino, $\neq$ Pantaleo Davide Cozzoli, ${ }^{\star,}, \neq$ and \\ Liberato Manna ${ }^{\ddagger}$ \\ Scuola Superiore ISUFI, University of Salento, Distretto Tecnologico ISUFI, via per Arnesano \\ km 5, I-73100 Lecce, Italy, National Nanotechnology Laboratory of CNR-INFM, Unità di \\ Ricerca IIT, via per Arnesano km 5, I-73100 Lecce, Italy, Laboratoire de Physique et de Chimie \\ des Nanoobjets-INSA, 135 Avenue de Rangueil, F-31077 Toulouse, France, CNR-Istituto di \\ Cristallografia (IC), via Amendola 122/O, I-70126, Bari, Italy, Dipartimento di Fisica "A. \\ Volta”, Università di Pavia, and Unità CNR-INFM and CNISM, via Bassi 6, I-27100, Pavia, \\ Italy, and Istituto di Fisiologia Generale e Chimica Biologica "G. Esposito", Milano and \\ S3-CNR-INFM, Modena, Italy
}

Received October 19, 2007; E-mail: albert.figuerola@qi.ub.es; davide.cozzoli@unile.it

\begin{abstract}
A one-pot, two-step colloidal strategy to prepare bimagnetic hybrid nanocrystals (HNCs), comprising size-tuned fcc FePt and inverse spinel cubic iron oxide domains epitaxially arranged in a heterodimer configuration, is described. The HNCs have been synthesized in a unique surfactant environment by temperature-driven sequential reactions, involving the homogeneous nucleation of $\mathrm{FePt}$ seeds and the subsequent heterogeneous growth of iron oxide. This self-regulated mechanism offers high versatility in the control of the geometric features of the resulting heterostructures, circumventing the use of more elaborate seeded growth techniques. It has been found that, as a consequence of the exchange coupling between the two materials, the HNCs exhibit tunable single-phase-like magnetic behavior, distinct from that of their individual components. In addition, the potential of the heterodimers as effective contrast agents for magnetic resonance imaging techniques has been examined.
\end{abstract}

\section{Introduction}

The availability of colloidal nanocrystals (NCs) with sizeand shape-dependent physical-chemical properties has opened new technological horizons in nanoengineering, nanoelectronics, and nanomedicine. ${ }^{1-6}$ As compared to other classes of nanostructured materials, NCs offer unique advantages in terms of ease of fabrication, postsynthesis processability, and relatively straightforward implementation in both devices and biological environments.

During the past decade, a high level of advancement in surfactant-assisted synthetic procedures has been achieved, allowing for a fine control over both the size and shape for a

\footnotetext{
University of Salento.

† Unità di Ricerca IIT.

$\S$ Laboratoire de Physique et de Chimie des Nanoobjets-INSA.

" CNR-IC.

$\perp$ Università di Pavia and Unità CNR-INFM and CNISM.

\# Istituto di Fisiologia Generale e Chimica Biologica "G. Esposito".

(1) Eustis, S.; El-Sayed, M. A. Chem. Soc. Rev. 2006, 35, 209-217.

(2) Burda, C.; Chen, X.; Narayanan, R.; El-Sayed, M. A. Chem. Rev. 2005, $105,1025-1102$.

(3) Rosi, N. L.; Mirkin, C. A. Chem. Rev. 2005, 105, 1547-1562.

(4) Pellegrino, T.; Kudera, S.; Liedl, T.; Muñoz Javier, A.; Manna, L.; Parak, W. J. Small 2005, 1, 48-63.

(5) Jun, Y.-W.; Jang, J.-T.; Cheon, J. Bull. Korean Chem. Soc. 2006, 27, 961971.

(6) Euliss, L. E.; DuPont, J. A.; Gratton, S.; DeSimone, J. Chem. Soc. Rev. 2006, 35, 1095-1104
}

10.1021/ja078034v CCC: $\$ 40.75$ @ 2008 American Chemical Society variety of metal, semiconductor, and oxide NCs. ${ }^{7-11}$ Recently, first prototypes of more sophisticated colloidal NCs have been also devised, in which domains of different materials with individually tailored geometric parameters are joined through epitaxial interfaces, providing individually processable composite particles. ${ }^{12}$ Such all-inorganic nano-objects with a topologically controlled distribution of their composition, henceforth referred to as hybrid nanocrystals (HNCs), offer a higher technological potential over that of conventional NCs based on a single inorganic material. Indeed, HNCs represent robust multifunctional entities, in which optically, magnetically, and catalytically active sections can coexist without the need of bridging molecules or embedding matrixes. In addition, because of the overlapping of the electronic bands of adjacent material domains, HNCs can exhibit modified or even unexpected physical-chemical behavior, which is difficult to attain both for any of their components alone and for their physical

(7) Lu, A.-H.; Salabas, E. L.; Schüth, F. Angew. Chem. Int. Ed. 2007, 46, $1222-1244$.

(8) Park, J.; Joo, J.; Kwon, S. G.; Jang, Y.; Hyeon, T. Angew. Chem., Int. Ed. 2007, 46, 4630-4660.

(9) Murphy, C. J.; Gole, A. M.; Hunyadi, S. E.; Orendorff, C. J. Inorg. Chem 2006, 45, 7544-7554.

(10) Jun, Y.-w.; Choi, J.-s.; Cheon, J. Angew. Chem., Int. Ed. 2006, 45, 34143439.

(11) Kumar, S.; Nann, T. Small 2006, 2, 316-329.

(12) Cozzoli, P. D.; Pellegrino, T.; Manna, L. Chem. Soc. Rev. 2006, 35, $1195-$ 1208. 
mixtures. As a further consequence, different chemical-physical properties may be exchange coupled, which makes HNCs promising for the realization of new generations of magnetooptoelectronic devices, such as spin-LEDs and spintransistors. ${ }^{13-15}$

Both kinetic processes and thermodynamic factors come into play during the synthesis of HNCs in colloidal media, ultimately deciding which topology the resulting nanoheterostructure will adopt.12 For example, core@shell HNCs, which combine different inorganic portions in an onionlike arrangement, can form when the involved materials have similar crystal structure and lattice parameters or when synthetic conditions allow interfacial strain to be compensated for by a decrease in surface energy. ${ }^{12}$ This configuration has been used to serve disparate purposes, such as to increase and/or tune plasmon absorption or photoluminescence, to modify magnetic properties, and to improve photocatalytic and photoelectrochemical responses, depending on the specific combinations. ${ }^{12,16-33}$

Materials that are poorly miscible and/or structurally dissimilar can be configured as nonconcentric heterostructures, such as heterodimers/oligomers, in which two or more inorganic domains with tailored sizes and/or shapes are interconnected through limited junction areas. In comparison with the core@shell geometry, this architecture is advantageous in that, in addition to enabling multifunctionality, it provides multiple surface platforms that can facilitate the site-specific anchoring of biomolecules, ${ }^{34,35}$ electrical connections, ${ }^{36}$ and chemically directed assembly of colloidal nanostructures. ${ }^{37}$ Thus far, the

(13) Li, Y.; Zhang, Q.; Nurmikko, A. V.; Sun, S. Nano Lett. 2005, 5, 16891692.

(14) Salazar-Alvarez, G.; Sort, J.; Suriñach, S.; Baró, M. D.; Nogués, J. J. Am. Chem. Soc. 2007, 129, 9102-9108.

(15) Li, K.; Wu, Y.; Guo, Z.; Zheng, Y.; Han, G.; Qiu, J.; Luo, P.; An, L.; Zhou, T. J. Nanosci. Natechnol. 2007, 7, 13-45.

(16) Manna, L.; Scher, E. C.; Li, L.-S.; Alivisatos, A. P. J. Am. Chem. Soc. 2002, 124, 7136-7145.

(17) Talapin, D. V.; Mekis, I.; Götzinger, S.; Kornowski, A.; Benson, O.; Weller, H. J. Phys. Chem. B 2004, 108, 18826-18831.

(18) Yu, K.; Zaman, B.; Romanova, S.; Wang, D.-s.; Ripmeester, J. A. Small 2005, 1, 332-338.

(19) Lu, W.; Wang, B.; Zeng, J.; Wang, X.; Zhang, S.; Hou, J. G. Langmuir $\mathbf{2 0 0 5}, 21,3684-3687$

(20) Cao, Y. W.; Jin, R.; Mirkin, C. A. J. Am. Chem. Soc. 2001, 123, 79617962.

(21) Lee, W. R.; Kim, M. G.; Choi, J. R.; Park, J. I.; Ko, S. J.; Oh, S. J.; Cheon, J. J. Am. Chem. Soc. 2005, 127, 16090-16097.

(22) Yang, J.; Lee, J. Y.; Too, H.-P. J. Phys. Chem. B 2005, 109, 1920819212.

(23) Tom, R. T.; Nair, A. S.; Singh, N.; Aslam, M.; Nagendra, C. L.; Philip, R.; Vijayamohanan, K.; Pradeep, T. Langmuir 2003, 19, 3439-3445.

(24) Hirakawa, T.; Kamat, P. V. J. Am. Chem. Soc. 2005, 127, 3928-3934

(25) Park, J.-I.; Cheon, J. J. Am. Chem. Soc. 2001, 123, 5743-5746.

(26) Srnová-Sloufová, I.; Vlcková, B.; Bastl, Z.; Hasslett, T. L. Langmuir 2004, 20, 3407-3415.

(27) Bao, Y.; Calderon, H.; Krishnan, K. M. J. Phvs. Chem. C 2007, 111, 19411944

(28) Teng, X.; Black, D.; Watkins, N. J.; Gao, Y.; Yang, H. Nano Lett. 2003, 3, 261-264.

(29) Zeng, H.; Li, J.; Wang, Z. L.; Liu, J. P.; Sun, S. Nano Lett. 2004, 4, $187-$ 190.

(30) Talapin, D. V.; Koeppe, R.; Gotzinger, S.; Kornowski, A.; Lupton, J. M.; Rogach, A. L.; Benson, O.; Feldmann, J.; Weller, H. Nano Lett. 2003, 3 , $1677-1681$.

(31) Carbone, L.; et al. Nano Lett. 2007, 7, 2942-2950.

(32) Talapin, D. V.; Nelson, J. H.; Shevchenko, E. V.; Aloni, S.; Sadtler, B.; Alivisatos, A. P. Nano Lett. 2007, 7, 2951-2959.

(33) Habas, S. E.; Lee, H.; Radmilovic, V.; Somorjai, G. A.; Yang, P. Nat. Mater. 2007, 6, 692-697.

(34) Gu, H.; Yang, Z.; Gao, J.; Chang, C. K.; Xu, B. J. Am. Chem. Soc. 2005, $127,34-35$

(35) Choi, J.-s.; Jun, Y.-w.; Yeon, S.-I.; Kim, H. C.; Shin, J.-S.; Cheon, J. J. Am. Chem. Soc. 2006, 128, 15982-15983.

(36) Mokari, T.; Rothenberg, E.; Popov, I.; Costi, R.; Banin, U. Science 2004 304, 1787-1790.

(37) Salant, A.; Amitay-Sadovsky, E.; Banin, U. J. Am. Chem. Soc. 2006, 128, 10006-10007. synthesis of oligomer-like HNCs has been accomplished by various approaches, most of which have been generally based on seeded growth techniques. In this regard, mechanisms as diverse as the thermally activated coalescence of an initially deposited shell, ${ }^{38}$ catalyzed reactions at liquid-liquid interfaces, ${ }^{34}$ heterogeneous nucleation, ${ }^{35,38-43}$ or controlled fusion of preformed particles ${ }^{43,44}$ have been exploited, allowing magnetic, metallic, and fluorescent spherical sections to be combined into binary (such as of $\mathrm{FePt}-\mathrm{CdS}(\mathrm{Se}),{ }^{38,39} \gamma-\mathrm{Fe}_{2} \mathrm{O}_{3}-$ metal sulfide, ${ }^{45} \mathrm{FePt}-\mathrm{Ag}, \mathrm{Fe}_{3} \mathrm{O}_{4}-\mathrm{Ag},{ }^{34} \mathrm{Au}-\mathrm{Fe}_{3} \mathrm{O}_{4},{ }^{40} \mathrm{CoPt}_{3}-$ $\mathrm{Au}^{41}$ and $\mathrm{FePt}-\mathrm{Au}{ }^{35}$ and ternary HNCs (such as of Au$\mathrm{Fe}_{3} \mathrm{O}_{4}-\mathrm{Au}, \mathrm{PbS}-\mathrm{Au}-\mathrm{PbS}, \mathrm{Fe}_{3} \mathrm{O}_{4}-\mathrm{Au}-\mathrm{PbSe},{ }^{42,43}$ and $\mathrm{Pd}-$ Co-Pd sulfide).$^{44}$ Further developments have demonstrated the possibility to grow foreign material sections onto selected locations of anisotropically shaped NCs. Examples include CdS-, $\mathrm{CdSe}-$, and CdTe-based heterostructures with linear or branched topology, ${ }^{46}$ "nanodumbbells" and "nanomatchsticks" based on $\mathrm{CdSe}-\mathrm{Au},{ }^{36,47} \mathrm{CdS}-\mathrm{Au}^{48,49}$ and $\mathrm{CdS}-\mathrm{PbSe},{ }^{50} \mathrm{ZnO}$ and $\mathrm{TiO}_{2}$ nanorods decorated with $\mathrm{Ag},{ }^{51} \gamma-\mathrm{Fe}_{2} \mathrm{O}_{3},{ }^{52}$ and Co spheres, ${ }^{53}$ respectively. As a major drawback, most of the involved synthesis procedures are complicated by the necessity of carrying out at least two distinct steps, namely the preparation and isolation of the starting NC seeds, and their subsequent reaction with the precursors necessary to build the additional material domains.

On the other hand, HNCs synthesized by one-pot methods, in which all the reagents required to grow the heterostructures are present since the beginning of the synthesis in the same surfactant mixture, are inherently difficult to devise and in fact have been rather rare. These include only $\mathrm{Co}-\mathrm{Pd}$ and $\mathrm{Cu}-\mathrm{In}$ sulfide HNCs, obtained by thermally induced phase segregation of an initial material alloy, ${ }^{54,55}$ and symmetric $\mathrm{Ag}-\mathrm{AgBr}$ heterodimers, derived from simultaneous formation of the two

(38) Gu, H.; Zheng, R.; Zhang, X.; Xu, B. J. Am. Chem. Soc. 2004, 126, 56645665

(39) Gao, J. Z. B.; Gao, Y.; Pan, Y.; Zhang, X.; Xu, B. J. Am. Chem. Soc. 2007, 129, 11928-11935.

(40) Yu, H.; Chen, M.; Rice, P. M.; Wang, S. X.; White, R. L.; Sun, S. Nano Lett. 2005, 5, 379-382.

(41) Pellegrino, T.; Fiore, A.; Carlino, E.; Giannini, C.; Cozzoli, P. D.; Ciccarella, G.; Respaud, M.; Palmirotta, L.; Cingolani, R.; Manna, L. J. Am. Chem. Soc. 2006, 128, 6690-6698.

(42) Shi, W.; Zeng, H.; Sahoo, Y.; Ohulchanskyy, T. Y.; Ding, Y.; Wang, Z L.; Swihart, M.; Prasad, P. N. Nano Lett. 2006, 6, 875-881.

(43) Shi, W.; Sahoo, Y.; Zeng, H.; Ding, Y.; Swihart, M. T.; Prasad, P. N. Adv. Mater. 2006, 18, 1889-1894.

(44) Teranishi, T.; Saruyama, M.; Nakaya, M.; Kanehara, M. Angew. Chem. Int. Ed. 2007, 46, 1713-1715.

(45) Kwon, K.-W.; Shim, M. J. Am. Chem. Soc. 2005, 127, 10269-10275.

(46) Milliron, D. J.; Hughes, S. M.; Cui, Y.; Manna, L.; Li, J.; Wang, L.-W.; Alivisatos, A. P. Nature 2004, 430, 190-195.

(47) Mokari, T.; Sztrum, C. G.; Salant, A.; Rabani, E.; Banin, U. Nat. Mater. 2005, 4, 855-863.

(48) Carbone, L.; Kudera, S.; Giannini, C.; Ciccarella, G.; Cingolani, R.; Cozzoli, P. D.; Manna, L. J. Mater. Chem. 2006, 16, 3952-3956.

(49) Saunders, A. E.; Popov, I.; Banin, U. J. Phys. Chem. B 2006, 110, $25421-$ 25429.

(50) Kudera, S.; Carbone, L.; Casula, M. F.; Cingolani, R.; Falqui, A.; Snoeck, E.; Parak, W. J.; Manna, L. Nano Lett. 2005, 5, 445-449.

(51) Pacholski, C.; Kornowski, A.; Weller, H. Angew. Chem., Int. Ed. 2004, 43, 4774-4777

(52) Buonsanti, R.; Grillo, V.; Carlino, E.; Giannini, C.; Curri, M. L.; Innocenti, C.; Sangregorio, C.; Achterhold, K.; Parak, F. G.; Agostiano, A.; Cozzoli, P. D. J. Am. Chem. Soc. 2006, 128, 16953-16970.

(53) Casavola, M.; Grillo, V.; Carlino, E.; Giannini, C.; Gozzo, F.; Fernandez Pinel, E.; Garcia, M. A.; Manna, L.; Cingolani, R.; Cozzoli, P. D. Nano Lett. 2007, 7, 1386-1395.

(54) Teranishi, T.; Inoue, Y.; Nakaya, M.; Oumi, Y.; Sano, T. J. Am. Chem. Soc. 2004, 126, 9914-9915.

(55) Choi, S.-H.; Kim, E.-G.; Hyeon, T. J. Am. Chem. Soc. 2006, 128, 25202521 . 
inorganic portions. ${ }^{56}$ It appears clear that the development of such types of colloidal strategies, in which both homogeneous and heterogeneous nucleation/growth processes of different materials can be controlled at once, would be highly convenient to simplify synthetic procedures to HNCs. Nevertheless, to date this target remains yet an extremely challenging task.

The present work represents an advance in this respect, since it reports on a novel one-pot, two-step colloidal approach to prepare bimagnetic HNCs based on size-tunable fcc FePt and inverse spinel cubic iron oxide domains arranged in a heterodimer configuration. The HNCs have been synthesized in a unique surfactant environment by temperature-driven sequential reactions, involving the homogeneous nucleation of FePt seeds and the subsequent heterogeneous growth of iron oxide. This self-regulated mechanism offers high versatility in the control of the geometric features of the resulting heterostructures. A combination of techniques has been used to provide an exhaustive morphological, structural, and magnetic characterization of the HNCs. The heterodimers have been also dissolved in water by a polymer-coating procedure, ${ }^{60}$ and their potential as $T_{2}$-relaxing contrast agents for magnetic resonance imaging (MRI) has been comparatively examined with respect to the clinically used Endorem contrasting agent. ${ }^{57}$

\section{Experimental Section}

Chemicals. Iron pentacarbonyl $\left(\mathrm{Fe}(\mathrm{CO})_{5}, 98 \%\right)$, oleic acid $\left(\mathrm{C}_{17} \mathrm{H}_{33-}\right.$ $\mathrm{CO}_{2} \mathrm{H}$ or OLAC, $\left.90 \%\right)$, oleyl amine $\left(\mathrm{C}_{17} \mathrm{H}_{33} \mathrm{NH}_{2}\right.$ or OLAM, $\left.70 \%\right)$, and 1-octadecene $\left(\mathrm{C}_{18} \mathrm{H}_{36}\right.$ or ODE, $\left.90 \%\right)$ were purchased from Aldrich. Platinum(II) acetylacetonate $\left(\mathrm{Pt}(\mathrm{acac})_{2}, 99 \%\right)$ was purchased from Strem. OLAC, OLAM, and ODE were degassed under vacuum and stored under nitrogen in a glovebox at $-20^{\circ} \mathrm{C}$ before use. All syntheses were carried out under air-free conditions, using a standard Schlenk line setup.

Synthesis of FePt Nanocrystals. FePt NCs were prepared with sizes ranging from 3 to $9 \mathrm{~nm}$ by slightly modified literature procedures. ${ }^{58,59}$ These methods relied on the high-temperature reduction of a platinum(II) salt with $\mathrm{Fe}(\mathrm{CO})_{5}$ in an ODE solution containing OLAC and OLAM surfactants at $170-200{ }^{\circ} \mathrm{C}$. Details on the synthesis can be found in Section 1 of the Supporting Information.

Synthesis of FePt-Iron Oxide Hybrid Nanocrystals. To synthesize $\mathrm{FePt}$-iron oxide heterodimer $\mathrm{HNCs}$, the crude reaction mixture containing the FePt NCs that had formed upon heating at $170-200{ }^{\circ} \mathrm{C}$ for $1 \mathrm{~h}$ (as described in the previous paragraph) was further heated to $295{ }^{\circ} \mathrm{C}$ (under $\mathrm{N}_{2}$ ) at a rate of approximately $8^{\circ} \mathrm{C} / \mathrm{min}$ and kept at this temperature for an additional $1 \mathrm{~h}$. Under these circumstances, thermal decomposition of excess iron oleate complexes in the solution led to the generation of iron oxide. After this period, the flask was allowed to cool to room temperature, after which it was opened to air, and the HNCs were precipitated upon adding 2-propanol, separated by centrifugation, and finally redissolved in either hexane, toluene, or chloroform. The temporal evolution of HNC growth was monitored by extracting small aliquots of the hot reaction mixture via a syringe at scheduled time intervals. The aliquots were suddenly cooled, and the NCs were purified as described above.

Solubilization of FePt Seeds and HNCs into Water. To perform magnetic relaxivity measurements, water-soluble samples were required.

(56) Saunders, A. E.; Popov, I.; Banin, U. Z. Anorg. Allg. Chem. 2007, 633, 2414-2419

(57) Wang, Y.-X. J.; Hussain, S. M.; Krestin, G. P. Eur. Radiol. 2001, 11, 23192331 .

(58) Chen, M.; Liu, J. P.; Sun, S. J. Am. Chem. Soc. 2004, 126, 8394-8395.

(59) Chen, M.; Kim, J.; Liu, J. P.; Fan, H.; Sun, S. J. Am. Chem. Soc. 2006, 128, 7132-7133.

(60) Pellegrino, T.; Manna, L.; Kudera, S.; Liedl, T.; Koktysh, D.; Rogach, A. L.; Keller, S.; Rädler, J.; Natile, G.; Parak, W. J. Nano Lett. 2004, 4, 703707.

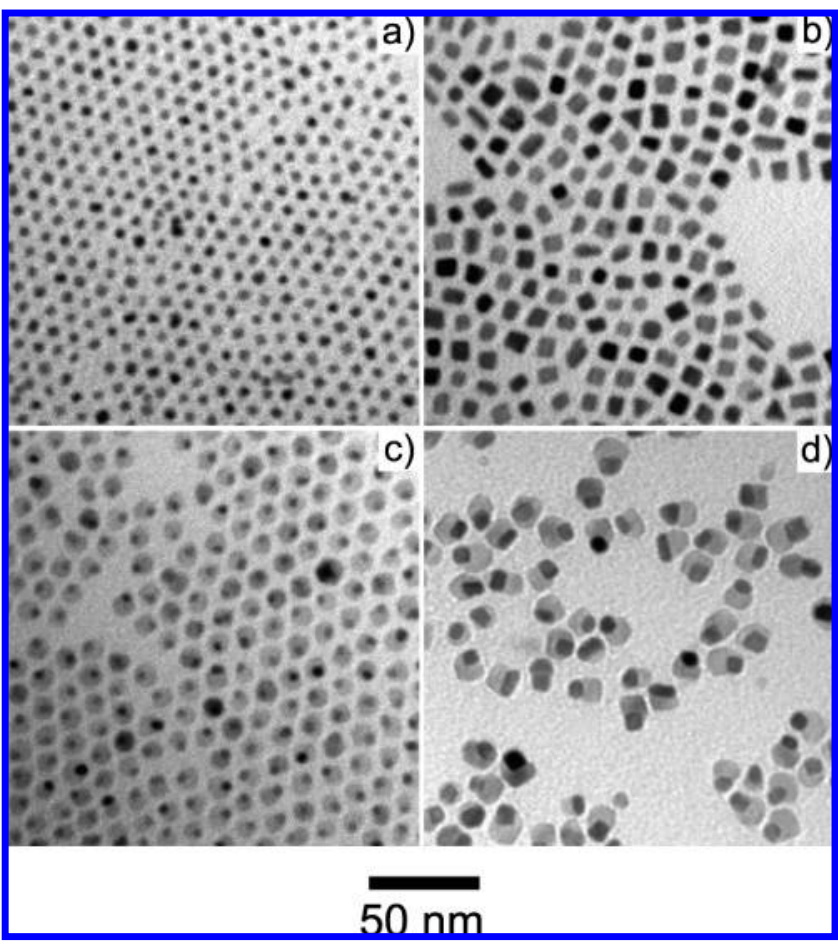

Figure 1. TEM images of nanocrystal products synthesized at a $\mathrm{Fe}(\mathrm{CO})_{5} /$ $\mathrm{Pt}(\mathrm{acac})_{2}$ ratio of 4 (see Table 1 for further details). (a, b) FePt seeds extracted after being heated for $1 \mathrm{~h}$ at $200^{\circ} \mathrm{C}$. (c, d) FePt-iron oxide HNCs obtained from the previous FePt seeds upon further heating at $295{ }^{\circ} \mathrm{C}$ for $1 \mathrm{~h}$.

To this aim, we applied a previously developed procedure that relied on coating the FePt seeds/HNCs with an amphiphilic polymer shell, which provided them with full solubility in aqueous media while preventing aggregation. ${ }^{60}$

Powder X-ray Diffraction (XRD). XRD measurements were performed with a NONIUS KappaCCD single-crystal diffractometer equipped with a $3 \mathrm{~kW}$ generator molybdenum tube, a high-precision four-circle goniometer, and a low-noise and high-sensitivity CCD detector. The samples were measured in Debye-Scherrer configuration using Lindemann capillaries $(0.5$ - $\mathrm{mm}$ diameter) that were filled with a dried NC powder. The images were folded into powder diffraction patterns using the FIT2D software ${ }^{61}$ after calibrating the detector with Si-NIST (640c) powder standard.

Transmission Electron Microscopy (TEM). Low-magnification TEM images were recorded with a Jeol Jem 1011 microscope operating at an accelerating voltage of $100 \mathrm{kV}$. Phase-contrast high-resolution TEM (HRTEM) measurements were performed with a Jeol $2100 \mathrm{~F}$ microscope, equipped with a field emission gun and working at the accelerating voltage of $200 \mathrm{kV}$. The samples for analysis were prepared by dropping a dilute solution of the HNCs in hexane on carbon-coated copper grids and then allowing the solvent to evaporate. A statistical analysis was carried out on several wide-field, low-magnification TEM images, with the help of a dedicated software (Image J 200). For each sample, 200 particles were counted at least.

Compositional Analysis. An inductively coupled plasma atomic emission spectrometer (ICP-AES) Varian Vista AX was used to measure the Fe and Pt content of purified FePt seeds/HNCs. The samples were dissolved in concentrated $\mathrm{HCl} / \mathrm{HNO}_{3}(3 / 1 \mathrm{v} / \mathrm{v})$ and digested by using a CEM "MARS 5" microwave digester.

Magnetic Measurements. Magnetic measurements were carried out on dried nanocrystal powder samples using a Quantum Design SQUID MPMS-XL7 magnetometer. Zero-field-cooled (ZFC) and field-cooled

(61) Cervellino, A.; Giannini, C.; Guagliardi, A.; Ladisa, M. Phys. Rev. B 2005, 72,35412 . 
Table 1. Synthetic Parameters Used for the Preparation of FePt-Iron Oxide HNCs Reported in Figures 1 and 2

\begin{tabular}{|c|c|c|c|c|c|c|c|c|}
\hline $\begin{array}{c}\mathrm{Fe} / \mathrm{Pt} \\
\text { molar } \\
\text { ratio }\end{array}$ & $\begin{array}{l}\mathrm{Pt}(\mathrm{acac})_{2} \\
(\mathrm{mg})\end{array}$ & $\begin{array}{c}\mathrm{Fe}(\mathrm{CO})_{5} \\
(\mathrm{mg})\end{array}$ & $\begin{array}{l}\text { OLAC } \\
(\mathrm{mg})\end{array}$ & $\begin{array}{l}\text { OLAM } \\
(\mathrm{mg})\end{array}$ & $\begin{array}{c}(\mathrm{OLAM}+\mathrm{OLAC}) / \mathrm{Pt} \\
\text { molar ratio }\end{array}$ & $\begin{array}{l}\text { ODE } \\
(\mathrm{mL})\end{array}$ & $\begin{array}{l}d_{\text {FePt }} \\
(n m)\end{array}$ & $\begin{array}{c}d_{\mathrm{Fe}-\mathrm{oxide}} \\
(\mathrm{nm})\end{array}$ \\
\hline \multirow[t]{2}{*}{4} & 50 & 95 & 785 & 955 & 20 & 7.5 & $8.9 \pm 1.1$ & $12.0 \pm 1.5$ \\
\hline & 300 & 597 & 785 & 955 & 3.3 & 7.5 & $4.4 \pm 0.6$ & $9.5 \pm 0.9$ \\
\hline \multirow[t]{2}{*}{12} & 50 & 286 & 785 & 955 & 20 & 7.5 & $6.1 \pm 0.7$ & $15.0 \pm 1.6$ \\
\hline & 100 & 570 & 785 & 955 & 10 & 7.5 & $4.0 \pm 0.5$ & $11.1 \pm 1.2$ \\
\hline
\end{tabular}

(FC) curves were recorded after cooling the samples in the absence or in the presence of a 50 Oe field, respectively, in the $2-300 \mathrm{~K}$ range. Field-dependent magnetization measurements were recorded between -5 and $5 \mathrm{~T}$ both at $5 \mathrm{~K}$ and at room temperature.

$T_{1} / T_{2}$ Relaxation Measurements. ${ }^{1} \mathrm{H}$ nuclear magnetic resonance (NMR) relaxometry characterization was performed by measuring the longitudinal and the transverse nuclear relaxation times, $T_{1}$ and $T_{2}$, respectively, in the 5-212 $\mathrm{MHz}$ frequency range, which corresponds to an external magnetic field spanning from 0.15 to $5 \mathrm{~T}$. A Bruker electromagnet and an Oxford 0-9 $\mathrm{T}$ superconducting magnet were used to generate the static magnetic field. The range was selected to cover most of the frequencies used by MRI commercial tomographs dedicated to clinical use and/or laboratory research. The measurements were performed at room temperature. The differences in $T_{1}$ and $T_{2}$ with respect to the values obtained at physiological temperature were within $10 \%$. The NMR signal generation and detection were obtained by using a Bruker MSL200 spectrometer and a Tecmag Apollo pulsed FT-NMR spectrometer. Standard radio frequency pulse excitation sequences based on the $\pi / 2-\pi$ Hahn echo technique were used. ${ }^{62}$ The $\pi / 2$ pulse length was selected to be between 1.5 and $17 \mu$ s, depending on the operating frequency of the spectrometer. The intensity of the radio frequency field $B_{1}$ was sufficiently strong to irradiate the whole NMR line, as the full width at half-maximum (fwhm) was in the range $0.2<$ fwhm $<7 \mathrm{kHz}$. The spin-lattice relaxation rate, $1 / T_{1}$, was obtained from the recovery of the longitudinal component of the nuclear magnetization following a short sequence of saturating radio frequency pulses.

\section{Results}

The synthesis of the $\mathrm{FePt}$-iron oxide heterodimers was carried out in a single reaction flask, whereby formation of $\mathrm{FePt}$ NCs (henceforth referred to as the "seeds") was followed by selective iron oxide deposition on such particles. The products isolated at different reaction stages were analyzed in detail, as described in the following sections.

3.1. Effect of the Geometric Features of the FePt Seeds on the Topology of the HNCs. The heating of the reaction mixture to $170-200{ }^{\circ} \mathrm{C}$ for $1 \mathrm{~h}$ induced the homogeneous nucleation and steady growth of FePt NCs. The absolute concentrations of the $\mathrm{Fe}$ and $\mathrm{Pt}$ organometallic precursors in the reaction vessel were the most influential parameters in controlling the dimensional and geometric features of the FePt seeds. In a set of experiments, the total reaction volume, the $\mathrm{Fe}(\mathrm{CO})_{5}$-to- $\mathrm{Pt}(\mathrm{acac})_{2}$ precursor molar ratio (which was set to 4), and the absolute amount of surfactants were kept constant, while the absolute amounts of Fe and Pt organometallic reagents were varied (the experimental details are summarized in Table 1). In general, it was noted that syntheses carried out at relatively higher Fe and Pt loadings led to the formation of FePt NCs with gradually smaller mean sizes. In Figure 1a,b, representative low-magnification TEM overviews are shown for FePt NCs synthesized at an $\mathrm{Fe}(\mathrm{CO})_{5} / \mathrm{Pt}(\mathrm{acac})_{2}$ molar ratio of 4 . The images reveal that the NCs that grew larger $(\geq 5-6 \mathrm{~nm})$ developed a

(62) Abragam, A. Principles of Nuclear Magnetism; Clarendon: Oxford, 1961. pronounced faceting (mainly cube-shaped objects were observable along with a minor fraction of triangular-like, rodlike, and other irregularly shaped particles). As opposed, the smaller NCs $(\leq 5 \mathrm{~nm})$ exhibited a nearly spherical morphology.

Following the formation of the FePt NCs, further heating of the reaction mixtures to $295{ }^{\circ} \mathrm{C}$ for an additional $1 \mathrm{~h}$ led to $\mathrm{FePt}$-iron oxide heterodimers with different relative dimensions of the metallic and oxide domains. Figure 1c,d reports TEM images of HNCs that derived from the same FePt seeds previously shown in panels a and $b$, respectively. The dimensional features of the HNCs are summarized in Table 1. Since iron oxide exhibited a comparatively lower image contrast than FePt, the low-magnification TEM images provided preliminary information about the relative topological distribution of the two material domains in the final HNCs. Heterogeneous deposition of iron oxide on relatively smaller FePt seeds provided them with an incomplete and spatially asymmetric shell, which conferred the resulting heterodimers a markedly peanutlike profile (Figure 1c). These heterostructures appeared to expose only a rather limited area of the FePt surface to the external environment. On the other hand, on the larger faceted FePt seeds the iron oxide domains were preferentially localized on a few selected facets, so that the final HNCs resembled a dumbbelllike shape with a more symmetric spatial distribution of the two material sections (Figure 1d). In the latter type of HNC configuration, a more extended fraction of the original FePt seed surface was left uncovered by the oxide.

3.2. Effect of the Fe-to-Pt Precursor Molar Ratio. Systematic experiments highlighted that $\mathrm{Fe}(\mathrm{CO})_{5} / \mathrm{Pt}(\mathrm{acac})_{2}$ molar ratios remarkably lower than 4 were insufficient to guarantee the generation of FePt-iron oxide HNCs in high yields. The effect of increasing the relative amounts of the organometallic precursor in the reaction mixture was therefore studied. As a representative case, TEM images corresponding to syntheses carried out at a Fe/Pt molar ratio of 12 and at different values of the absolute Fe and Pt reactant concentrations are shown in Figure 2 (see Table 1 for details). Panels a and $\mathrm{b}$ indicate that HNCs were already formed at $200{ }^{\circ} \mathrm{C}$. In particular, several small iron oxide patches could be observed all over the surface of the smaller FePt seeds (panel a). Differently, only one or eventually two iron oxide domains nucleated on selected facets of the larger and faceted FePt seeds (panel b). These results clearly proved that under reaction conditions that supplied a large excess of iron oleate complexes, the heterogeneous nucleation of iron oxide could be anticipated to the lowtemperature stage. Nevertheless, it was noted that a decrease in the reaction temperature down to $170-180{ }^{\circ} \mathrm{C}$ could retard or even prevent the oxide deposition, ultimately yielding FePt NCs with proportionally increased sizes (Section 2 and Figure S1 in the Supporting Information). These facts allowed us to infer that the growth of the FePt seeds proceeded until iron oxide 


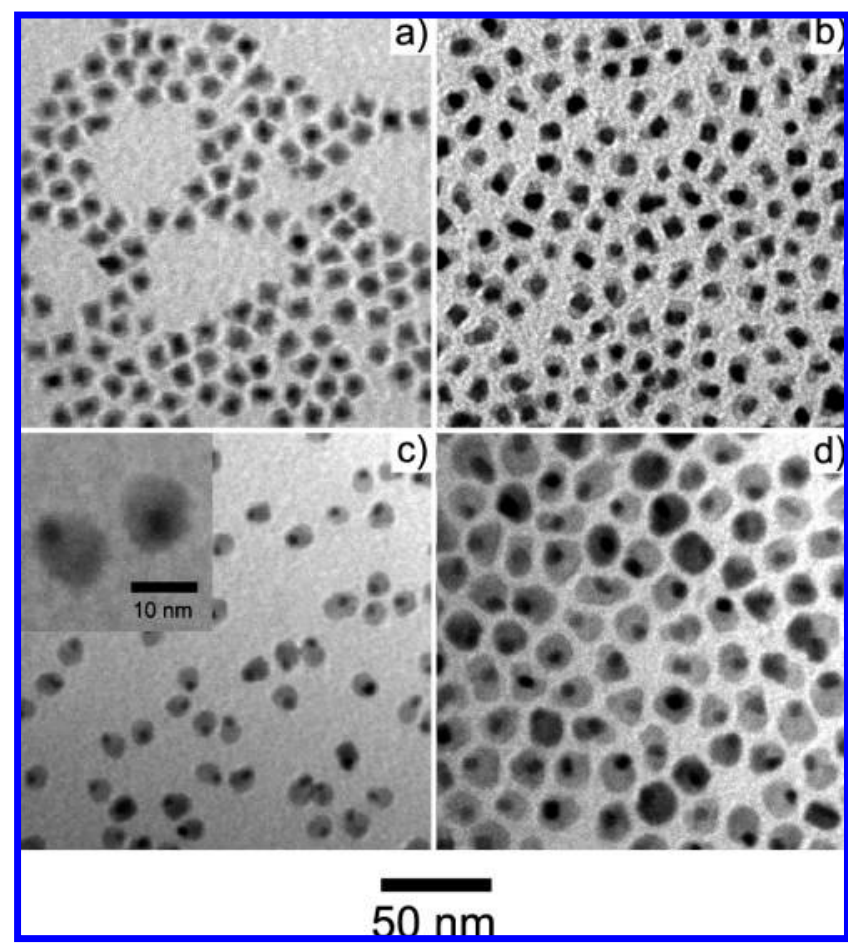

Figure 2. TEM images of nanocrystal products synthesized at a $\mathrm{Fe}(\mathrm{CO})_{5} /$ $\mathrm{Pt}(\mathrm{acac})_{2}$ ratio of 12 (see Table 1 for further details). (a, b) FePt-iron oxide HNCs extracted after heating for $1 \mathrm{~h}$ at $200{ }^{\circ} \mathrm{C}$. (c, d) FePt-iron oxide HNCs obtained from the previous heterostructures upon subsequent heating at $295{ }^{\circ} \mathrm{C}$ for $1 \mathrm{~h}$.

formed on their surfaces, whereby it inhibited further enlargement of the FePt domains to a significant extent. Upon prolonged heating at $295^{\circ} \mathrm{C}$, a peculiar structural evolution was detected. The iron oxide patches that had initially formed at $200{ }^{\circ} \mathrm{C}$ grew further, while progressively coalescing into a single iron oxide section located at one side of the original FePt seeds (more details on such time-dependent topological changes are reported in Section 3 and Figure S2 of the Supporting Information). Following this process, the final heterostructures generally exhibited a highly asymmetric peanutlike profile (Figure $2 \mathrm{c}, \mathrm{d}$, respectively).

3.3. Compositional Analysis. The $\mathrm{Pt} / \mathrm{Fe}$ molar ratios in $\mathrm{FePt}$ seeds (properly isolated at either 170 or $200{ }^{\circ} \mathrm{C}$ before occurrence of iron oxide overgrowth) and therefrom-derived FePt-iron oxide HNCs were determined by ICP analysis (Section 4 and Table S1 in the Supporting Information). Concerning the size evolution of $\mathrm{Fe}_{x} \mathrm{Pt}_{100-x}$ composition, a significant increase in the relative Pt content was measured for FePt seeds at increasing dimensions ( $x$ ranged from 70 to 15 ), in agreement with previous studies and mechanistic interpretations (as discussed in Section 4 of the Supporting Information). ${ }^{58,59,63}$ The $\mathrm{Pt} / \mathrm{Fe}$ ratios measured for the HNCs were in all cases lower than the values obtained for their corresponding FePt seeds, which was fully consistent with the presence of the additional iron oxide domain.

3.4. High-Resolution TEM Analysis. HRTEM investigations provided more detailed information on the structure of the HNCs. As described above, core@shell-type heterostructures with often flowerlike morphology were formed at the early stages of iron oxide deposition. These objects could carry either

(63) Nandwana, V.; Elkins, K. E.; Poudyal, N.; Chaubey, G. S.; Yano, K.; Liu, J. P. J. Phys. Chem. C 2007, 111, 4185-4189.

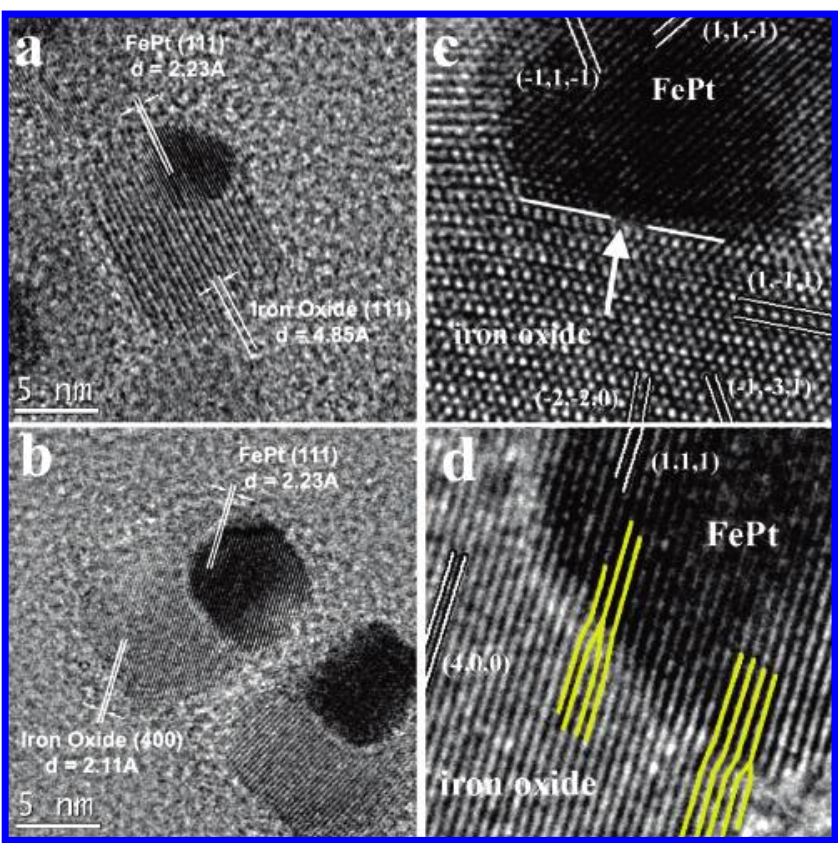

Figure 3. HRTEM study of individual FePt-iron oxide heterodimers with different topologies. (a, b) Lattice relationships between relevant sets of planes of the respective lattices. (c, d) Magnified views of interfacial areas between the two material domains. (c) An arrow indicates point defects located in between two regions (marked by white lines) corresponding to slightly deformed boundary planes. (d) Dislocations are marked in yellow.

single-crystalline or polycrystalline shells (Section 5 and Figures S3 and S4 in the Supporting Information), as reported previously. ${ }^{29}$ Here, we will focus on the structure of the fully grown heterodimers, where each of the starting FePt seeds is ultimately connected to a single iron oxide domain. Figure 3 reports a representative HRTEM study on HNCs with different heterodimer topology and relative dimensions of the component domains. The images clearly demonstrate a high crystallinity across the entire heterostructure. In agreement with XRD measurements (Section 6 and Figure S5 in the Supporting Information), fast Fourier transform analyses confirmed that in each HNC one domain was made of disordered fcc FePt, while the other one was composed of inverse spinel cubic iron oxide (i.e., $\mathrm{Fe}_{3} \mathrm{O}_{4}$ and/or $\gamma-\mathrm{Fe}_{2} \mathrm{O}_{3}$, which are indistinguishable from each other to the extent of HRTEM). Lattice distances that correspond to characteristic planes of the respective phases could be clearly identified.

A few types of heterointerface configurations have been encountered more frequently. Figure 3a shows the HRTEM image of an asymmetric HNC in which an iron oxide portion surrounds a small and nearly spherical FePt domain almost entirely. The respective material lattices are mutually arranged such that the $111_{\mathrm{FePt}}$ and the $111_{\mathrm{Fe}-\text { oxide }}$ planes are aligned parallel to each other. This ensures the continuity of atomic fringes on the corresponding orthogonal direction, where a lattice distance given by the sum of two consecutive $111_{\mathrm{FePt}}$ planes (that equals $2 d_{111}=4.46 \AA$ ) indeed projects regularly to one $111_{\mathrm{Fe}-\text { oxide }}$ spacing $\left(d_{111}=4.85 \AA\right)$. According to the coincidence site lattice theory, the mismatch associated with this one-dimensional interface would be of about $8 \% .{ }^{64}$ Figure $3 \mathrm{~b}$ reports an HRTEM analysis of a more symmetric HNC. Under the observed zone

(64) Randle, V. The Role of the Coincidence Site Lattice in Grain Boundary Engineering; Woodhead Publishing: Cambridge, England, 1997. 


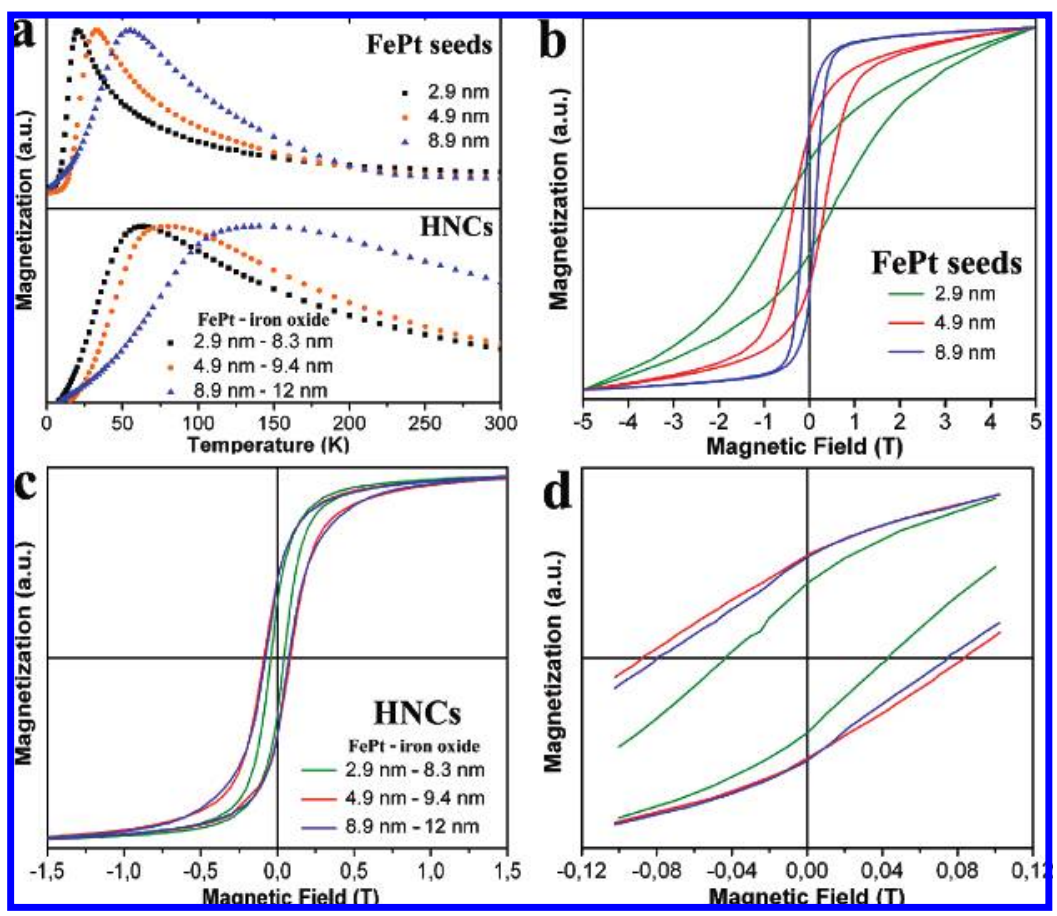

Figure 4. (a) ZFC curves for differently sized FePt seeds and therefrom-derived HNCs. (b) Normalized hysteresis loops measured at $5 \mathrm{~K}$ for the same FePt seeds. (c) Corresponding loops for therefrom-prepared HNCs. (d) Zoom of panel c in the low field region.

Table 2. Magnetic Parameters Measured for Differently Sized FePt Seeds and Therefrom-Synthesized FePt-Iron Oxide HNCs, Corresponding to Data in Figure 4

\begin{tabular}{|c|c|c|c|c|c|c|}
\hline \multicolumn{4}{|c|}{ FePt seeds } & \multicolumn{3}{|c|}{ FePt-iron oxide HNCs } \\
\hline $\begin{array}{l}d_{\text {Fept }} \\
(n m)\end{array}$ & $\begin{array}{l}\mathrm{Fe}_{x} \mathrm{Pt}_{100-x} \\
(\text { ICP) }\end{array}$ & $\begin{array}{l}T_{\mathrm{B}} \\
(\mathrm{K})\end{array}$ & $\begin{array}{l}H_{c} \\
\text { (G) }\end{array}$ & $\begin{array}{c}d_{\mathrm{Fe}-0 \times \mathrm{ide}} \\
(\mathrm{nm})\end{array}$ & $\begin{array}{l}T_{\mathrm{B}} \\
(\mathrm{K})\end{array}$ & $\begin{array}{l}H_{c} \\
\text { (G) }\end{array}$ \\
\hline $2.9 \pm 0.6$ & $\mathrm{Fe}_{70} \mathrm{Pt}_{30}$ & 20 & 5700 & $8.3 \pm 1.0$ & 64 & 435 \\
\hline $4.9 \pm 0.7$ & $\mathrm{Fe}_{39} \mathrm{Pt}_{61}$ & 32 & 3600 & $9.4 \pm 1.2$ & 80 & 900 \\
\hline $8.9 \pm 1.1$ & $\mathrm{Fe}_{15} \mathrm{Pt}_{85}$ & 55 & 1330 & $12.0 \pm 1.5$ & 140 & 800 \\
\hline
\end{tabular}

axis, each $111_{\mathrm{FePt}}$ plane $\left(d_{111}=2.23 \AA\right)$ coincides with one $400_{\mathrm{Fe}-\text { oxide }}$ plane $\left(d_{400}=2.11 \AA\right)$, resulting in a smaller lattice misfit of $5.4 \%{ }^{64}$ In general, the magnified views of the border regions between the two material domains in a heterodimer revealed the occurrence of crystal imperfections at the interfaces, such as point or linear defects, induced by the lattice misfit. For example, in panel c a slight deformation of the lattice plane at the boundary between the FePt and iron oxide domains is evidenced by two white segments. As a consequence of such distortion, point crystal defects are present in correspondence of the zone indicated by the arrow. Furthermore, in panel d, a progressive misalignment of the lattice planes can be observed in different interface regions, where it ultimately leads to edge dislocations.

3.5. SQUID Magnetic Characterization. The study of the transition from the superparamagnetic to the ferromagnetic regime was performed by FC and ZFC measurements. ${ }^{65,66}$ The $\mathrm{ZFC}$ measurements are reported in Figure 4, while the relevant parameters are summarized in Table 2. For the initial FePt seeds (top of Figure $4 \mathrm{a}$ ), the blocking temperature, $T_{\mathrm{B}}$ (corresponding to the maximum in the respective $\mathrm{ZFC}$ curves), increased with increasing mean size, $d_{\mathrm{FePt}}$, as found previously. ${ }^{67,68}$ In the case

(65) Jeong, U.; Teng, X.; Wang, Y.; Yang, H.; Xia, Y. Adv. Mater. 2007, 19, $33-60$

(66) Sun, S. Adv. Mater. 2006, 18, 393-403.

(67) Sun, S.; Murray, C. B.; Weller, D.; Folks, L.; Moser, A. Science 2000, 287, 1989-1992. of the hybrid systems (bottom of Figure 4a), systematically higher $T_{\mathrm{B}}$ values were measured, with the net differences in $T_{\mathrm{B}}$ being roughly proportional to the size of the iron oxide domain, $d_{\mathrm{Fe}-\text { oxide, }}$ attained in the respective cases (Table 2). The fielddependent hysteresis loops (Figure $4 \mathrm{~b}-\mathrm{d}$ ) confirm the ZFC results. Indeed, all samples were superparamagnetic at room temperature, as authenticated by both zero coercive field $\left(H_{\mathrm{c}}\right)$ and residual magnetization $\left(M_{\mathrm{r}}\right)$, while they exhibited a ferromagnetic behavior below $T_{\mathrm{B}}$. Notably, the $H_{\mathrm{c}}$ values measured at $5 \mathrm{~K}$ for the initial $\mathrm{FePt}$ seeds decreased monotonically with increasing $d_{\mathrm{FePt}}$ (Figure $4 \mathrm{~b}$ ), which was in agreement with the reduction in magnetocrystalline anisotropy that is associated with the relative $\mathrm{Pt}$ enrichment in the seed composition (Table 2). ${ }^{58,59,63,65-71}$ These $H_{\mathrm{c}}$ values were further diminished following the growth of iron oxide domain (panels $\mathrm{c}$ and $\mathrm{d}$ ). A more careful analysis suggested that the coercive fields were better correlated to the ratio between the sizes of the respective domains, $d_{\mathrm{Fe}-\text { oxide }} / d_{\mathrm{FePt}}$, rather than to their absolute values, as previously found for their core@ shell counterparts. ${ }^{29}$ As a matter

(68) Nakaya, M.; Kanehara, M.; Teranishi, T. Langmuir 2006, 22, 3485-3487.

(69) Elkins, K. E.; Vedantam, T. S.; Liu, J. P.; Zeng, H.; Sun, S.; Ding, Y. Wang, Z. L. Nano Lett. 2003, 3, 1647-1649.

(70) Sun, S.; Anders, S.; Thomson, T.; Baglin, J. E. E.; Toney, M. F.; Hamann H. F.; Murray, C. B.; Terris, B. D. J. Phys. Chem. B 2003, 103, 54195425 .

(71) Howard, L. E. M.; Nguyen, H. L.; Giblin, S. R.; Tanner, B. K.; Terry, I.; Hughes, A. K.; Evans, J. S. O. J. Am. Chem. Soc. 2005, 127, 1014010141. 
of fact, the HNCs became magnetically harder when they adopted roughly symmetric configurations $\left(d_{\mathrm{Fe}-\text { oxide }} / d_{\mathrm{FePt}} \approx\right.$ $1-2)$, while they were softer in the case of spatially asymmetric topologies $\left(d_{\mathrm{Fe}-\text { oxide }} / d_{\mathrm{FePt}} \approx 2.5-3\right)$.

3.6. Relaxivity Studies. One interesting application of superparamagnetic NCs concerns their ability to enhance the image contrast in MRI techniques by modifying the proton relaxation rates in different tissues. The NCs induce magnetic field inhomogeneities in the surrounding medium that significantly decrease the transverse relaxation time $\left(T_{2}\right)$ of the protons. The shortening in $T_{2}$ leads to a signal loss and, in turn, to negatively contrasted images. We have therefore explored the present FePt-iron oxide HNCs as MRI contrast agents.

The NMR dispersion (NMRD) profile allows one to measure the frequency dependence of the longitudinal $R_{1}$ and transverse $R_{2}$ nuclear relaxivities. ${ }^{72}$ For a given target nuclear species (in most cases the proton), these quantities are defined as:

$$
R_{i}=\left(-1 /\left.T_{i}\right|_{\text {dia }}+1 /\left.T_{i}\right|_{\text {meas }}\right) / C \quad i=1,2
$$

where $C$ is the concentration of the magnetic atoms $(\mathrm{Fe}), 1 /\left.T_{\mathrm{i}}\right|_{\text {dia }}$ is the relaxation rate of the proton nuclei in the diamagnetic host, and $1 /\left.T_{\mathrm{i}}\right|_{\text {meas }}$ is the nuclear relaxation rate measured in the presence of the superparamagnetic fluid. We performed roomtemperature NMRD measurements on FePt NCs and corresponding $\mathrm{FePt}$-iron oxide $\mathrm{HNCs}$ at varying concentrations. For this purpose, the NC samples were fully dissolved in water by using a previously developed polymer-coating procedure ${ }^{60}$ that preserved the nanoparticles from aggregation in aqueous media (Figure S6 in the Supporting Information). Figure 5 reports the longitudinal $R_{1}$ and transverse $R_{2}$ relaxivities (panels a and b, respectively) as a function of the frequency for four FePt-iron oxide HNC samples with different dimensional features. The commercial compound, known as Endorem contrasting agent, was used as the reference. As normally observed for superparamagnetic nanoparticles, the HNCs mainly behaved as $T_{2^{-}}$ relaxing MRI contrast agents. Indeed, the effects of $\mathrm{HNC}$ dimensions and geometry on their longitudinal relaxivity $\left(R_{1}\right)$ were quite small, as inferred from Figure 5a. On the other hand, a variation in the proton transverse relaxivity as a function of the HNC dimensions was clearly observed. The NMRD data in Figure $5 \mathrm{~b}$ revealed that the $R_{2}$ values for each sample remained constant over the whole frequency range investigated, showing a profile similar to that recorded for Endorem contrasting agent. However, significant differences among the absolute $R_{2}$ values were detected. Indeed, compared to Endorem contrasting agent, sample $\mathrm{B}\left(d_{\mathrm{FePt}}=4.0 \mathrm{~nm}, d_{\mathrm{Fe}-\text { oxide }}=11.1 \mathrm{~nm}\right)$ showed lower $R_{2}$, while samples $\mathrm{C}\left(d_{\mathrm{FePt}}=6.2 \mathrm{~nm}, d_{\mathrm{Fe}-\text { oxide }}=\right.$ $15.4 \mathrm{~nm})$ and $\mathrm{D}\left(d_{\mathrm{FePt}}=8.9 \mathrm{~nm}, d_{\mathrm{Fe}-\text { oxide }}=12.0 \mathrm{~nm}\right)$ showed slightly higher and lower values, respectively. Interestingly, for sample A $\left(d_{\mathrm{FePt}}=10.0 \mathrm{~nm}, d_{\mathrm{Fe}-\text { oxide }}=16.0 \mathrm{~nm}\right), R_{2}$ increased by a factor of almost two at any frequency. From these results, it emerges that the proton relaxation rate scales up with the overall dimensions of HNCs, so that an improvement with respect to Endorem contrasting agent can be achieved for heterodimers larger than $\sim 20 \mathrm{~nm}$. For comparison purposes, NMRD measurements were also performed on the corresponding FePt seeds (Figure S7 in the Supporting Information). In

(72) Muller, R. N. In Encyclopedia of Nuclear Magnetic Resonance; Grant, D. M., Harris, R. K., Eds.; Wiley: New York, 1996; pp 1438-1444.

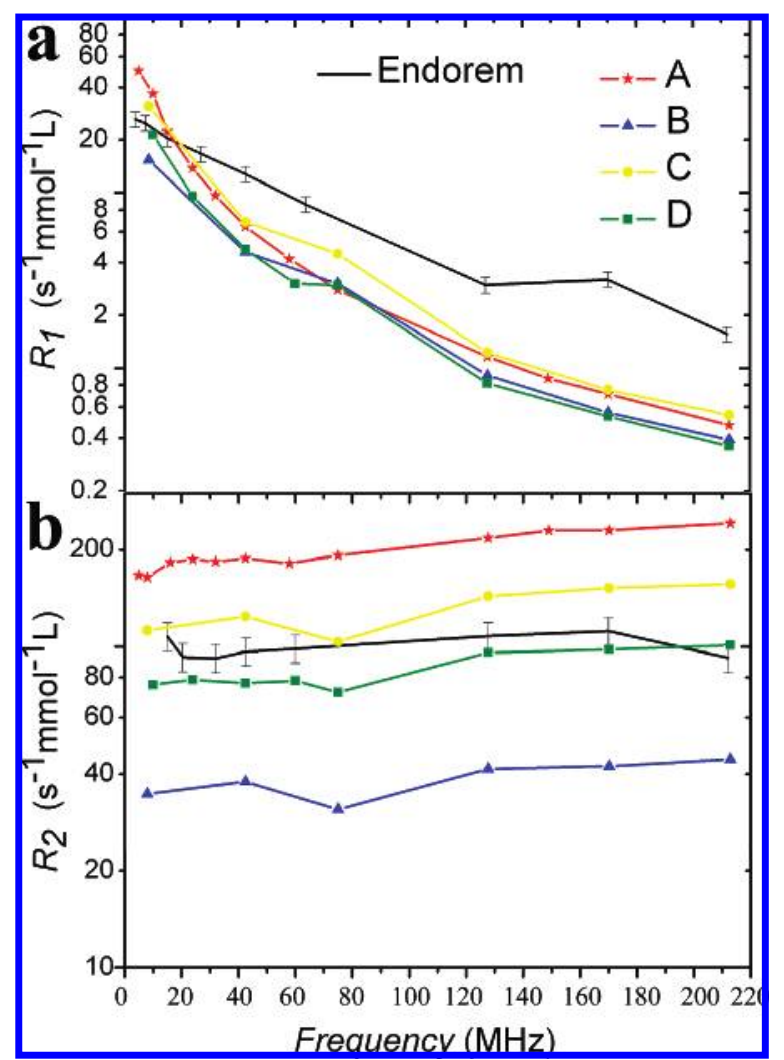

Figure 5. Plots of room-temperature ${ }^{1} \mathrm{H}$ NMRD relaxivities $R_{1}$ (a) and $R_{2}$ (b) vs frequency for the various HNC samples dissolved in water. The FePt and iron oxide domain dimensions were, respectively: (A) 10.0 and 16.0 $\mathrm{nm}$, (B) 4.0 and $11.1 \mathrm{~nm},(C) 6.2$ and $15.4 \mathrm{~nm}$, (D) 8.9 and $12.0 \mathrm{~nm}$. For a comparison, the relaxivity values for Endorem contrasting agent are also shown.

this case, the observed relaxivities were remarkably lower than those measured for the corresponding HNCs and did not show any clear dependence on the NC size.

\section{Discussion}

In this work, a one-pot, two-step colloidal strategy has been devised to synthesize bimagnetic NC-based heterostructures, each comprising one fcc cubic FePt domain and one inverse spinel cubic iron oxide portion epitaxially arranged in a dimerlike configuration. The HNCs have been prepared in a unique solution environment via two sequential reactions, sketched in Scheme 1, involving the initial homogeneous nucleation of FePt NCs at $T \leq 200{ }^{\circ} \mathrm{C}$, followed by the heterogeneous growth of iron oxide onto those seeds at $T \approx 295{ }^{\circ} \mathrm{C}$. Since each reaction stage is selectively activated under well-defined thermal conditions, then regulation of both the temperature and the heating time guarantees that the two material sections of the HNCs form at distinct times of the synthesis course. Such level of control allows for the high-yield preparation of FePt-iron oxide heterodimer-like HNCs with the respective domain sizes being tunable independently. Therefore, the present scheme naturally circumvents major drawbacks of conventional seed-mediated approaches to NC-based heterostructures, in which the seeds of a starting material need to be first prepared and purified in an independent step and subsequently introduced into a different surfactant environment to react with the precursors of an additional material. 
Scheme 1. Mechanism of the Temperature-Controlled Formation of FePt-Iron Oxide Heterodimers

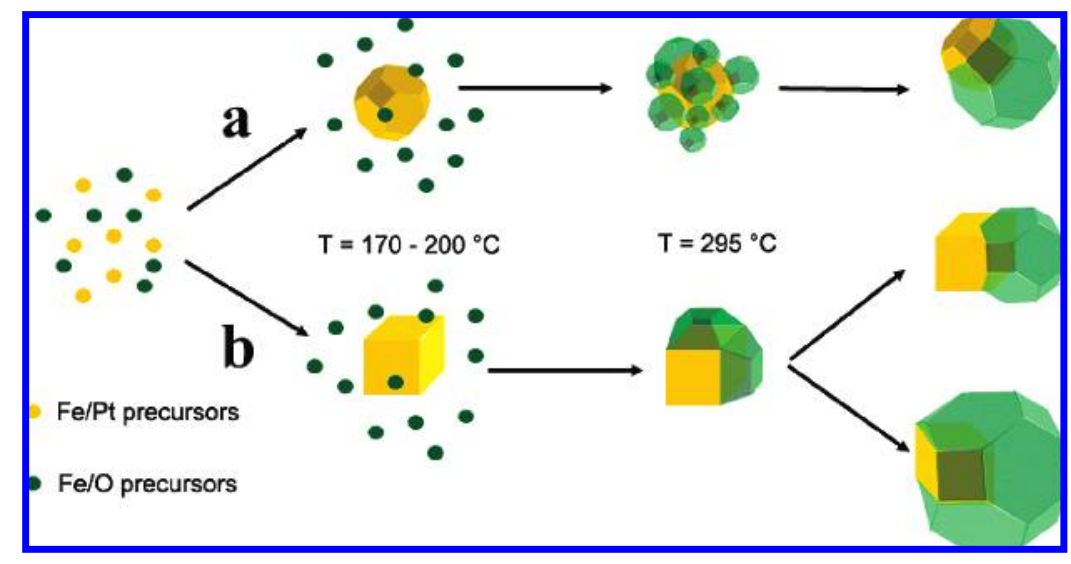

The synthesis of the FePt-iron oxide heterodimers is performed in the presence of equimolar amounts of OLAC and OLAM surfactants, which have been previously used to grow nanosized FePt and iron oxide NCs and their core@shell combinations. ${ }^{29,58,59,73-76}$ Here, the chemistry involved in the two individual colloidal systems is suitably combined and manipulated to achieve novel hybrid architectures. Upon fast ligand exchange, both $\mathrm{Pt}$-surfactant and $\mathrm{Fe}$-surfactant complexes are formed in the solution. ${ }^{59,73}$ While the former mainly initiate and sustain FePt growth, ${ }^{59}$ the latter play multiple roles along the entire synthesis course, which therefore justifies the necessity of a large $\mathrm{Fe}(\mathrm{CO})_{5}$ excess in the reaction medium. Indeed, during FePt seed formation in the low-temperature stage (at $T \leq 200{ }^{\circ} \mathrm{C}$ ), $\mathrm{Fe}(0)$ - surfactant species not only represent a primary source of metallic $\mathrm{Fe}(0)$, but also are sacrificed in the reduction of the $\mathrm{Pt}(\mathrm{acac})_{2}$ precursor to $\mathrm{Pt}(0){ }^{58,59}$ In the hightemperature stage at $T=295^{\circ} \mathrm{C}$, substantial supply of $\mathrm{Fe}(\mathrm{II}) /$ $\mathrm{Fe}(\mathrm{III})-$ OLAC complexes is needed to sustain nucleation and growth of iron oxide upon thermal decomposition. ${ }^{73-76}$ OLAC and OLAM, on the other hand, assist in controlling the size and shape of both the FePt and the iron oxide domains.

The effects of the reaction parameters involved in the control of the geometrical features of the HNCs have been explored through systematic experiments, allowing us to gain an insight into the formation mechanism of the heterostructures.

4.1. Size Control and Chemical Composition of the FePt Seeds. Size-controlled fcc-FePt NCs have been reported by using different synthetic routes, such as, for instance, diol-,${ }^{67-69}$ hydride-- ${ }^{70}$ and $\mathrm{Fe}(\mathrm{CO})_{5}$-assisted reduction, ${ }^{58,59,63}$ and by exploiting a reduced iron precursor. ${ }^{71}$ In our syntheses, the mean size of the FePt NCs could be enlarged by adjusting the surfactant-to-Fe/Pt precursors molar ratio to gradually higher levels. This trend suggests that particularly stable surfactantmetal complexes are formed in a solution that dictate the relative balance of FePt nucleation and growth. ${ }^{63,77}$ The higher the

(73) Hyeon, T.; Lee, S. S.; Park, J.; Chung, Y.; Bin Na, H. J. Am. Chem. Soc. 2001, 123, 12798-12801.

(74) Park, J.; An, K.; Hwang, Y.; Park, J.-G.; Noh, H.-J.; Kim, J.-Y.; Park, J.-H.; Hwang, N.-M.; Hyeon, T. Nat. Mater. 2004, 3, 891-895.

(75) Park, J.; Lee, E.; Hwang, N.-M.; Kang, M.; Kim, S. C.; Hwang, Y.; Park, J.-G.; Noh, H.-J.; Kim, J.-Y.; Park, J.-H.; Hyeon, T. Angew. Chem., Int. Ed. 2005, 44, 2872-2877.

(76) Casula, M. F.; Jun, Y.-w.; Zaziski, D. J.; Chan, E. M.; Corrias, A.; Alivisatos, A. P. J. Am. Chem. Soc. 2006, 128, 1675-1682.

(77) Shevchenko, E. V.; Talapin, D. V.; Schnablegger, H.; Kornowski, A.; Festin, Ö.; Svedlindh, P.; Haase, M.; Weller, H. J. Am. Chem. Soc. 2003, 125, 9090-9101. surfactant concentration is in the solution, the lower is the number of nuclei that are initially generated, and consequently, the greater is the concentration of the metal active species left to feed enlargement of such nuclei. This explanation is indeed in agreement with the size trend found by us. The shape and compositional evolution of the $\mathrm{Fe}_{x} \mathrm{Pt}_{100-x} \mathrm{NCs}$ can be understood in light of the kinetically controlled growth mechanism recently proposed by Chen et al., ${ }^{59}$ in which the technique of sequentially injecting the OLAC and OLAM ligands has a crucial role (more details can be found in the Supporting Information).

4.2. Formation Mechanism of the FePt-Iron Oxide HNCs. Several pieces of evidence allowed us to identify the mechanism by which the FePt-iron oxide HNCs are formed under our experimental conditions. First, when the synthesis was carried out at $200{ }^{\circ} \mathrm{C}$ in the absence of $\mathrm{Pt}(\mathrm{acac})_{2}$ (which prevented the formation of FePt seeds), tiny $(<3 \mathrm{~nm})$ iron oxide NCs were obtained only after rather long reaction times $(>2 \mathrm{~h})$. On the other hand, the heating of the same mixtures to $295^{\circ} \mathrm{C}$ caused the growth of much larger $(30-100 \mathrm{~nm})$ and highly irregularly shaped iron oxide NCs. By comparison, iron oxide deposition on preformed $\mathrm{FePt}$ seeds was already identifiable after relatively shorter times at less than $200{ }^{\circ} \mathrm{C}$ (depending on the absolute $\mathrm{Fe}(\mathrm{CO})_{5}$ concentration), hence under milder temperatures than those required to grow pure iron oxide NCs in similar surfactant mixtures. These control experiments highlighted that the in situ nucleated FePt NCs not only acted as seed catalysts that facilitated the nucleation of iron oxide, but also dictated the geometric features of the iron oxide domains that were incorporated in the heterostructures.

Second, the monitoring of the standard heterostructure syntheses did not reveal any transient contamination of the HNC population with pure iron oxide NCs. This finding allows us to exclude the hypothesis that HNC formation could proceed via oriented attachment of iron oxide NCs to FePt NCs which had nucleated independently. In fact, if such a mechanism were operative, one should detect the progressive increase in the population of HNCs at the expense of the population of the other two types of particles. Instead, the exclusive observation of heterodimer-like HNCs and the lack of other types of NC aggregates at all reaction times further discredit the occurrence of any fusion-based growth pathways. As a further confirmation, the heating of mixtures of FePt NCs and iron oxide $\mathrm{NCs}$ provided by separate syntheses never led to permanently fused NC heterodimers. 
On the basis of the above-reported experimental observations, it can be concluded that the mechanism by which the HNCs are synthesized is based on the temperature-controlled heterogeneous nucleation of iron oxide onto the surface of preformed FePt NC seeds (Scheme 1). The detailed pathways by which iron oxide forms can be better understood on the basis of the peculiar chemical reactivity of $\mathrm{Fe}(\mathrm{CO})_{5}$ in hot surfactant mixtures. In these media, iron oxide nucleation, which requires a threshold temperature of $200-220{ }^{\circ} \mathrm{C}$, is delayed until a sufficiently high concentration of reactive "monomers" accumulates in the solution upon slow pyrolysis of $\mathrm{Fe}$-carboxylate complexes. ${ }^{73-76}$ The nucleation self-arrests as the solution supersaturation is relieved, following which a comparatively much faster growth of the initially generated nuclei takes place. ${ }^{76}$ It turns out that a temporal separation between the nucleation and the growth stages is naturally achieved in colloidal iron oxide systems. An equivalent description of this dynamics is provided by the classical nucleation theory, according to which the activation energy for the generation of $\mathrm{NC}$ nuclei in solution (homogeneous nucleation) is much higher than the kinetic barrier for enlargement of pre-existing seeds (heterogeneous nucleation/ growth). ${ }^{78,79}$ Profiting from such a mechanism, seed-mediated strategies have been indeed developed for preparing monodisperse size-tailored iron oxide NCs, ${ }^{73,75,80}$ as well as for developing iron oxide-based HNCs. ${ }^{40,45,52,81,82}$

The above arguments are well suited to rationalize the outcome of our syntheses. Indeed, the FePt NCs, which have been produced in situ during the low-temperature stage, trigger selective heterogeneous nucleation and growth of iron oxide domains onto such NCs upon subsequent heating. Apparently, the presence of FePt seeds allows the kinetic barrier for the heterogeneous nucleation of iron oxide to be appreciably reduced. This contributes to relieve the solution saturation below the onset for homogeneous nucleation of separate iron oxide $\mathrm{NCs}$, thus guaranteeing high heterodimer yields in a rather broad range of experimental conditions.

The two most important parameters that control the relative domain dimensions of the HNCs are the size of the starting FePt $\mathrm{NCs}$ and the $\mathrm{Fe}(\mathrm{CO})_{5} / \mathrm{Pt}(\mathrm{acac})_{2}$ molar ratio used in the synthesis. The former dictates the ultimate surface area available for heterogeneous deposition, while the latter regulates the growth of the iron oxide domain. In general, the heterostructures with largest iron oxide domains are only obtained when large FePt seeds are formed in the growing mixture. In addition, the smallest iron oxide domains that can be grown have sizes that are comparable to those of the starting FePt seeds.

Detailed TEM analyses of the products of time-dependent syntheses shed more light on the growth mechanism of the HNCs. During HNC syntheses involving relatively smaller FePt NCs $(\leq 4 \mathrm{~nm})$, intermediate flowerlike HNCs were observed, in which FePt seeds were decorated with multiple iron oxide patches. Upon being heated at $295^{\circ} \mathrm{C}$, the latter developed into larger "petals" that progressively coalesced together, so that a single iron oxide domain was ultimately located at one side of

(78) Mullin, J. W. Crystallization, 3rd ed.; Butterworth-Heinemann: Oxford, 1997

(79) Markov, I. V. Crystal Growth for Beginners: Fundamentals of Nucleation, Crystal Growth, and Epitaxy; World Scientific: Singapore, 2003.

(80) Sun, S.; Zeng, H. J. Am. Chem. Soc. 2002, 124, 8204-8205.

(81) Kwon, K.-W.; Lee, B. H.; Shim, M. Chem. Mater. 2006, 18, 6357-6363.

(82) Selvan, S. T.; Patra, P. K.; Ang, C. Y.; Ying, J. Y. Angew. Chem., Int. Ed. 2007, 46, 2448-2452. each seed (Scheme 1, path a). This evolution points to a sort of dewetting process as the driving force for the formation of the heterodimer topology, as already observed in the synthesis of other NC heterostructures. ${ }^{38,39,45}$ However, as compared to what has been reported in these previous studies, here the iron oxide shell that initially grows on the FePt seeds is not amorphous but crystalline at all reaction stages, as actually verified by us (Figures S3 and S4 in the Supporting Information). The dewetting should be driven presumably by the tendency of the HNCs to reach a higher thermodynamic stability upon reducing the strain energy at the interface between the two materials, while paying a proportionally smaller cost of additional total surface energy. ${ }^{64,79}$ Following the coalescence process, the iron oxide domain attains a lower surface-to-volume ratio than that globally associated with its parent flowerlike nanostructure, and it shares a limited number of facets with the FePt domain, thus minimizing the overall interfacial area. ${ }^{12}$ Such a structural evolution toward a heterodimer-like configuration appears to be consistent with the comparatively harsher reaction conditions employed in our syntheses, relative to those required to generate their FePt@ $\mathrm{Fe}_{3} \mathrm{O}_{4}$ core@ shell counterparts. ${ }^{29}$ In our work, higher temperatures and longer reaction times can be expected indeed to supply sufficient thermal energy to promote shell coalescence into a segregated discrete domain.

By comparison, HNCs deriving from relatively larger FePt $\mathrm{NCs}(\geq 5 \mathrm{~nm}$ ) evolved according to a different pathway (Scheme 1 , path $b$ ). In this case, the nucleation of just one or eventually two iron oxide domains per seed was observed, which steadily increased their dimensions over time. Such a growth mode can be explained by considering the presence of well-developed facets on these seeds, some of which can be expected to exhibit indeed more pronounced chemical reactivity and/or allow for a better accommodation of interfacial strain. ${ }^{12,45}$

4.3. Heterodimer Structure. According to our structural analyses, the two domains in the heterodimers showed some preferences for which arrangement of the relative lattice orientations could be adopted. Indeed, depending on the sizes of the starting seeds and, in turn, on the asymmetry of the heterostructures, a few characteristic sets of FePt-iron oxide epitaxial relations were more frequently identified, which presumably should correspond to configurations that allowed for the best lattice fit between the two materials. On the other hand, there exist large differences in structure and in lattice parameters between the fcc FePt ( $a=b=c=3.816 \AA$ ) $)$ and the inverse spinel cubic iron oxide phase $(a=b=c=8.268$ $\AA / 8.3457 \AA$ for magnetite/maghemite), which could disfavor or even prohibit the attainment of coherent $\mathrm{FePt}$-iron oxide interfaces. ${ }^{79}$ However, in contrast to these expectations, our synthesis approach led to high yields of FePt-iron oxide heterodimers that could tolerate lattice mismatch values as large as $\sim 8 \%$. Our experimental results can be explained readily by considering the involvement of some mechanisms allowing strain energy to be decreased, as previously ascertained for other highly mismatched $\mathrm{NC}$ heterostructures. ${ }^{52}$ In this regard, it can be rationalized that two main pathways can concur to efficiently compensate for the $\mathrm{FePt}$-iron oxide junction strain during the formation of the HNCs: first, the occurrence of dislocations, as directly probed by us; second, the adhesion of surfactants on the respective material surfaces. Indeed, it is known that the total surface energy associated with a strained material domain 
epitaxially grown onto a mismatched substrate is given by the sum of the interfacial energy (i.e., the solid/solid tension between the involved materials) and of the surface energy (i.e., the solution/solid tension in the case of colloidal NCs). The former term can be reduced upon nucleation of dislocations which alleviate the high lattice-mismatch-related tension accumulated at the junction regions between the two materials. ${ }^{52,79}$ The latter term can be lowered a considerable extent by the surfactants adhering to the HNCs. ${ }^{79}$ As a result of the joint actions of these two mechanisms, the formation of the HNCs becomes extremely convenient since it is accompanied by a significant decrease in the total surface energy of the heterostructured system. This explanation is fairly consistent with our findings.

4.4. Magnetic Properties. As a general characteristic, the HNCs show a smooth variation of the magnetization as a function of the applied field (i.e., without sudden changes in the slope of the curves), which closely resembles the behavior of single-phase materials. This is indicative of an effective exchange-spring coupling between the two components, which allows their respective magnetization vectors to reverse cooperatively. ${ }^{15,83-85}$ The magnetic properties of the heterostructures are ultimately dictated by the dimensions of the two domains and by the size-dependent composition of the FePt component. ${ }^{58,59,63}$ It follows that an accurate control over these parameters by appropriate synthesis design enables fine modulation of the magnetic properties of the heterostructures. Another distinctive feature of the HNCs is that, despite their blocking temperature scales up with the overall heterostructure dimensions, nevertheless their coercitivity is strongly reduced below the values associated with their parent FePt seeds. The latter property appears to be dependent on the relative volumes of the respective domains. These findings overall suggest that the magnetic hardness and the blocking temperature of the HNCs can be tuned independently by engineering their geometric parameters (i.e., the FePt and iron oxide domain sizes) in a versatile way. Therefore, our bimagnetic HNCs offer an additional degree of freedom in the control of the overall magnetic behavior of nanocrystal heterostructures, which cannot be achieved by any of their individual components. ${ }^{15,29,66}$

The preliminary studies on the proton nuclear relaxation in the presence of the FePt-iron oxide HNCs have proven the possibility to reach relaxivity values comparable or even higher than those achievable with the commercial Endorem contrasting agent ferrofluid that has been already tested for clinical purposes. ${ }^{57}$ According to the NMRD profiles, the transverse relaxation becomes progressively faster with comparatively larger heterodimers, an effect that cannot be obtained with the sole FePt seeds. It follows that the enhanced performances of the HNCs should be put in relation with the iron oxide component in the heterostructures. Accordingly, the relative contrast enhancement exhibits a size dependence similar to that documented for pure iron oxide NCs. ${ }^{86}$ Nevertheless, other factors should be taken into account to rationalize the contrast agent ability of the HNCs in a more accurate way. For example,

(83) Skomski, R.; Coey, J. M. D. Phys. Rev. B 1993, 48, 15812-15816.

(84) Jiang, J. S.; Pearson, J. E.; Liu, Z. Y.; Kabius, B.; Trasobares, S.; Miller D. J.; Bader, S. D.; Lee, D. R.; Haskel, D.; Srajer, G.; Liu, J. P. J. Appl. Phys. 2005, 97, 10K 311 .

(85) Zambano, A. J.; Oguchi, H.; Takeuchi, I.; Choi, Y.; Jiang, J. S.; Liu, J. P.; Lofland, S. E.; Josell, D.; Bendersky, L. A. Phys. Rev. B 2007, 75, 144429.

(86) Jun, Y.-w.; Huh, Y.-M.; Choi, J.-s.; Lee, J.-H.; Song, H.-T.; Kim, S.; Yoon, S.; Kim, K.-S.; Shin, J.-S.; Suh, J.-S.; Cheon, J. J. Am. Chem. Soc. 2005, 127, 5732-5733. it is important to remark that the behavior of the longitudinal relaxation rate, $R_{1}$, is similar to that found for other known superparamagnetic materials (e.g., Endorem contrasting agent). For the latter, the Curie and Néel relaxations of the magnetization have been assumed to be the relevant proton relaxation pathways. ${ }^{87}$ For the present HNCs, it is therefore reasonable to expect that the magnetically harder FePt phase could contribute an extra magnetic moment, which could play an important role in determining their relaxivity behavior. Another factor that should be considered is the effect of the surface coating on the activity of the contrast agent. Our HNCs are protected with a polymer shell that ensures their full solubility in water without leading to detectable particle aggregation. This dispersibility condition accounts for the increase in the relaxivity values that we found for progressively larger HNCs, in good agreement with recently dedicated studies. ${ }^{91}$

\section{Conclusions}

We have demonstrated a convenient one-pot colloidal approach to prepare bimagnetic HNCs based on size-tunable FePt and iron oxide domains arranged in a heterodimer configuration. The distribution of the chemical composition in the heterostructures as well as the epitaxial arrangement of the two domains have been carefully ascertained. The heterodimers have been synthesized by a temperature-regulated synthetic scheme, whereby homogeneous nucleation of FePt seeds and heterogeneous growth of iron oxide proceed sequentially in a unique surfactant mixture. This mechanism is especially advantageous in that it avoids the separate preparation and the purification of nanocrystals of one material to seed the growth of the other one, while offering high versatility in the control of the geometric features of the resulting heterostructures. As a consequence of the intimate contact between FePt and iron oxide domains, the HNCs exhibit interesting magnetic properties, which are distinct from those of the individual components. For these reasons, these HNC heterostructures could usefully serve as model systems for both experimental and theoretical assessment of exchange-spring coupling effects between magnetically hard and soft phases, which thus far have been rather elusive in colloidal particles. ${ }^{15,83-85}$ Moreover, the possibility to exploit these new heterodimers to induce faster relaxation of proton nuclear spins has been preliminarily investigated, demonstrating their potential as even better performing MRI contrast agents than standard Endorem contrasting agent when their dimensions are suitably engineered. Further studies are currently underway to gain a deeper insight into the mechanisms of the proton relaxation dynamics in the presence of such systems.

We believe that these FePt-iron oxide heterodimers could find further interesting applications especially in the biomedical field. In addition to being useful for MRI diagnostic techniques, they could be simultaneously exploited for therapeutic purposes, such as for killing cancer cells, through magnetically induced heating (i.e., hyperthermia). ${ }^{8-90}$ In addition, the availability of

(87) Roch, A.; Muller, R. N.; Gillis, P. J. Chem. Phys. 1999, 110, 5403-5411. (88) Pankhurst, Q. A.; Connolly, J.; Jones, S. K.; Dobson, J. J. Phys. D: Appl. Phys. 2003, 36, R167-R181.

(89) Mornet, S.; Vasseur, S.; Grasset, F.; Duguet, E. J. Mater. Chem. 2004, 14, $2161-2175$.

(90) Maenosono, S.; Saita, S. IEEE Trans. Magn. 2006, 42, 1638-1642.

(91) Tromsdorf, U. I.; Bigall, N. C.; Kaul, M. G.; Bruns, O. T.; Nikolic, M. S.; Mollwitz, B.; Sperling, R. A.; Reimer, R.; Hohenberg, H.; Parak, W. J. Forster, S.; Beisiegel, U.; Adam, G.; Weller, H. Nano Lett. 2007, 7, 24222427. 
a dual surface platform in the heterodimers could potentially enable their site-specific functionalization with different fluorophores, receptors, or biomolecules, for targeting purposes ranging from multiplex imaging, drug delivery, and chemically directed assembly of the heterostructures both in solution and onto substrates.

Acknowledgment. We thank Joan Ribas (Grup d'Interaccions Magnètiques, Departament de Química Inorgànica, Universitat de Barcelona) and Núria Clos (Unitat de Mesures Magnètiques, Serveis Científics i Tècnics, Universitat de Barcelona) for performing magnetic measurements. The Italian projects PRIN (ContractNo. 2005039758) andFIRB (Contract No. RBLA03ER38), the European projects NoE MAGMANet (Contract No. NMP3-
CT-2005 515767) and SA-NANO (Contract No. STRP 013698) are also acknowledged.

Supporting Information Available: Details on the synthesis of the FePt NCs, discussion on the shape and compositional control of the FePt seeds, effect of the Fe/Pt precursor ratio and of temperature on the formation of the HNCs, time evolution of iron oxide heterogeneous growth, powder XRD analyses, HRTEM studies on core@shell HNCs at their early growth stages, TEM images of water-dissolved HNCs, relaxivity studies on FePt seed NCs, and complete ref 31. This material is available free of charge via the Internet at http://pubs.acs.org.

JA078034V 